\title{
The effect of tin substitution on the excitonic properties of two dimensional metal halide
} perovskites

\author{
Giulia Folpini, ${ }^{\dagger}$ Maurizia Palummo, ${ }^{*} \ddagger$ Daniele Cortecchia, ${ }^{\dagger}$ Luca Moretti, $₫$ Giulio \\ Cerullo," Annamaria Petrozza, ${ }^{\dagger}$ Giacomo Giorgi, ${ }^{*}, \S, \|$ and Ajay Ram Srimath
}

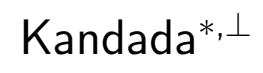

$\dagger$ †NST@Polimi, Istituto Italiano di Tecnologia,

Via Pascoli 70/3, Milano, Italy.

$\ddagger$ Dipartimento di Fisica and INFN, Universitá di Roma "Tor Vergata",

Via della Ricerca Scientifica 1 Roma, Italy

IDipartimento di Fisica, Politecnico di Milano,

Piazza Leonardo da Vinci 32, Milano, Italy.

$\S$ Department of Civil and Environmental Engineering (DICA), University of Perugia,

Via G. Duranti, 93 - 06125 - Perugia, Italy

\|CNR-SCITEC, I-06123, Perugia, Italy.

$\perp$ Department of Physics and Center for Functional Materials, Wake Forest University,

Winston-Salem (N.C), United States

E-mail: maurizia.palummo@roma2.infn.it; giacomo.giorgi@unipg.it; srimatar@wfu.edu

\begin{abstract}
With growing interest in the lead-free derivatives of the metal-halide perovskites, it is imperative to fully understand the contribution of the metal cation to their desir-
\end{abstract}


able excitonic characteristics. Here, we explore this question by performing an in-depth spectroscopic and theoretical analysis of phenethylammonium tin iodide $\left((\mathrm{PEA})_{2} \mathrm{SnI}_{4}\right)$, a prototypical tin based MHP, and rigorously compare it with its lead counterpart. We elaborate on the origin of multiple excitonic resonances uniquely observed in the linear absorption spectrum of (PEA) $)_{2} \mathrm{SnI}_{4}$ at energies about $200-300 \mathrm{meV}$ above the primary exciton. By performing calculations based on density functional theory and manybody perturbation theory, we suggest that the excitonic series at these higher energies are composed of electronic transitions from a lower lying valence band. Importantly, the valence band splitting is driven by the octahedral conformations that follow subtle variations in the organic-inorganic interactions within the crystal lattice. We experimentally show that the presence of the higher energy excitonic resonance results in a relatively slow nanosecond component in the formation dynamics of the primary exciton, in addition to the ultrafast phonon-driven hot carrier thermalization. While the presence of such slow relaxation channel for the excitons might be beneficial to many optoelectronic applications, our work suggests its possible control via systematic design of the organic cation. Moreover, our observations indicate that spin-orbit coupling does not play a primary role in the intricate yet crucial changes in the excitonic characteristics imparted by the tin substitution.

\section{Keywords}

Lead free perovskite, 2D excitons, exciton formation, exciton-phonon coupling

\section{Introduction}

The re-emergence of two-dimensional metal halide perovskites (2D MHPs) ${ }^{1-3}$ with large exciton binding energies has not only fueled technological interest, ${ }^{4,5}$ but also provided an excellent material platform to explore intriguing excitonic many-body physics ${ }^{6-11}$ via various advanced spectroscopies. From a technological standpoint, such spectroscopic studies are 
used to explore and determine the material handles that enable optimization of desirable optoelectronic properties - light emission, charge photo-generation and transport. ${ }^{12}$ To that end, most of the recent work on 2D-MHPs focused on the organic cation, highlighting its role in controlling the structural and vibrational parameters and consequently the electronic properties. ${ }^{13-15}$ Alternatively, mixing of two different organic cations has been suggested as a way to self-assemble 2D-MHPs of varied thicknesses with reduced defect densities and with excellent luminescence efficiencies. ${ }^{16-20}$

Most of the investigations, however, have been performed on lead-based systems, leaving the effect of the metal cation on the excitonic characteristics as an open question. This problem garners relevance due to the growing interest in tin-based ${ }^{21-31}$ and tin-alloyed ${ }^{32,33}$ MHPs driven by environmental and stability concerns caused by the use of lead. ${ }^{34}$ Substitution of lead with $\mathrm{Sn}^{2+35}$ or $\mathrm{Ge}^{2+33,36}$ will also enable us to address some of the contested claims on the peculiar characteristics of excitons in 2D-MHPs that distinguish them from their counterparts in other 2D materials. ${ }^{37}$ The imposing role of lattice dressing, the resultant polaronic effects, and the unique spin-structure due to the large spin-orbit coupling indeed are under-explored concepts in the context of 2D exciton physics. These issues can be addressed by a rigorous comparison of spectroscopic signatures of excitons in 2D-MHPs with comparable structural parameters, but with controlled substitution of the metal cation.

In this work, we perform steady-state and time-resolved spectroscopy of a prototypical 2D-MHP based on tin: phenethylammonium tin iodide $\left((\mathrm{PEA})_{2} \mathrm{SnI}_{4}\right)$ and compare its characteristics with those of phenethylammonium lead iodide $\left((\mathrm{PEA})_{2} \mathrm{PbI}_{4}\right)$, widely reported in the literature. In addition to the excitonic resonances well below the carrier continuum, we identify the spectroscopic signatures of higher energy excitonic states in $(\mathrm{PEA})_{2} \mathrm{SnI}_{4}$ which are absent in $(\mathrm{PEA})_{2} \mathrm{PbI}_{4}$. By using ground and excited-state ab-initio simulations, we explain the microscopic origin of the spectral features observed in the steady state optical absorption spectrum of $(\mathrm{PEA})_{2} \mathrm{SnI}_{4}$. Our analysis shows that, while the first optical resonance is due to a strongly bound exciton involving the two highest occupied and four 
lowest unoccupied states near the A-point of the Brillouin zone (BZ), the second optical peak around $2.3 \mathrm{eV}$ has a contribution from another excitonic series involving transitions from two lower-lying valence bands to the same set of unoccupied states. As we will discuss, the origin of these split valence bands is explained by the different structural distortion of the two inorganic layers induced by the organic components. Interestingly, although we demonstrate the similarity in the phonon driven, ultrafast carrier thermalization and exciton formation rates in (PEA $)_{2} \mathrm{SnI}_{4}$ and its lead counterpart, we clearly identify the presence of an additional exciton relaxation channel in the nanosecond regime owing to the higher lying excitonic states acting as population reservoirs for a part of the photo-generated carriers.

\section{Results}

The experimental absorption spectrum of (PEA) ${ }_{2} \mathrm{SnI}_{4}$ taken at $25 \mathrm{~K}$ is shown in Fig. 1(a). We observe a red-shift in the entire spectrum with respect to the lead counterpart (also shown in Fig. 1(a)), ${ }^{38}$ owing to the change in the lattice parameters, and a lineshape qualitatively similar to that of $(\mathrm{PEA})_{2} \mathrm{PbI}_{4}$ with a well defined excitonic resonance (labelled $X_{L E}$ ). Unlike in the case of $(\mathrm{PEA})_{2} \mathrm{PbI}_{4}$, here we do not observe a clear continuum edge and accordingly we cannot reproduce the lineshape using the Elliott's formalism. We note that the empirically modified Elliott's formalism used by Neutzner et al ${ }^{38,39}$ and Passarelli et al ${ }^{40}$ to reproduce the absorption spectrum of $(\mathrm{PEA})_{2} \mathrm{PbI}_{4}$ is also insufficient for the lineshape shown in Fig. 1(a). This highlights the need for a more rigorous analytical framework that considers the photophysical characteristics in their entirety beyond the empirical considerations, which is out of the scope of this work. 

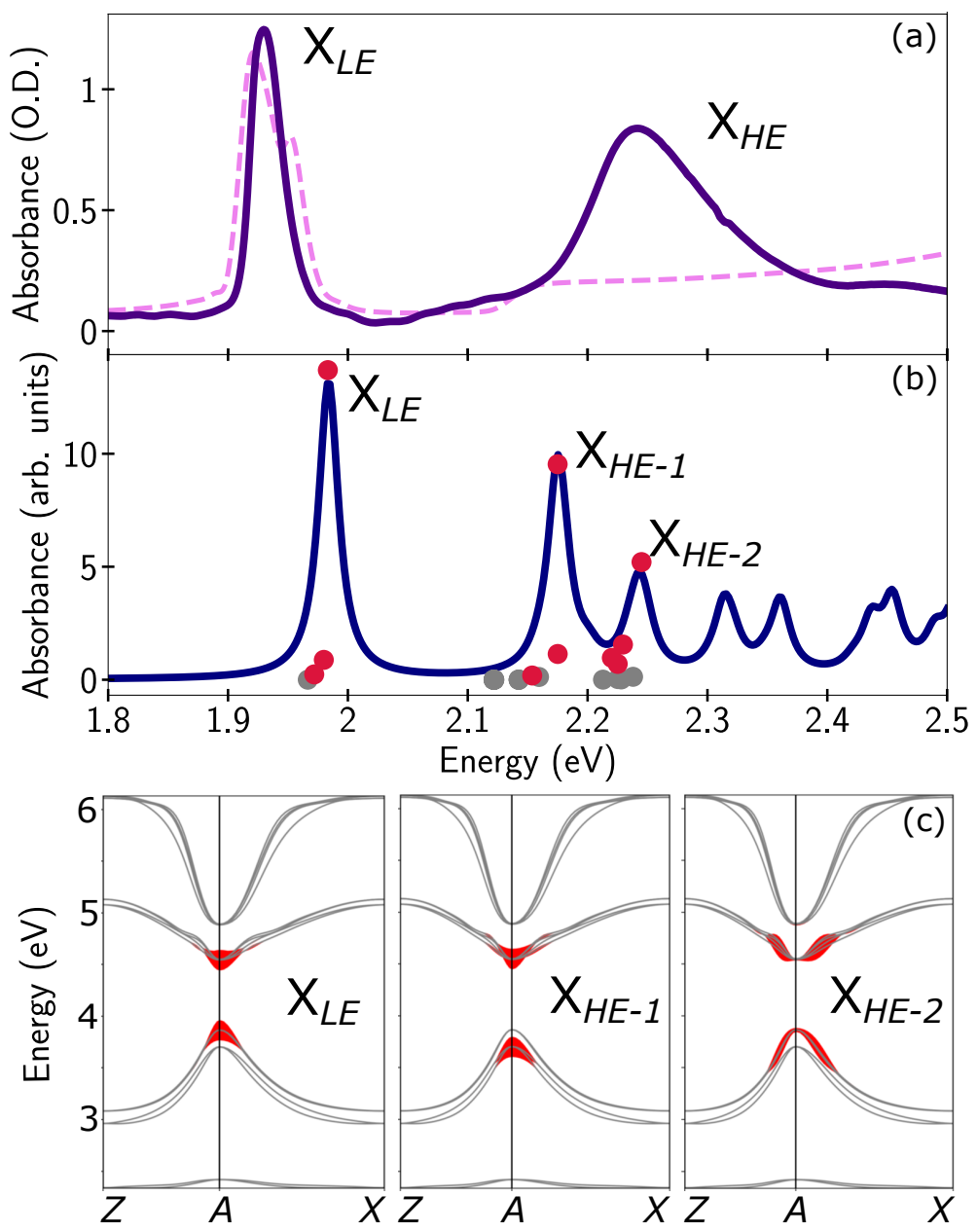

Figure 1: (a) Experimental linear absorption spectrum (solid line) of polycrystalline film of $(\mathrm{PEA})_{2} \mathrm{SnI}_{4}$ taken at $25 \mathrm{~K}$. The red-shifted (by $\left.440 \mathrm{meV}\right)$ spectrum of $(\mathrm{PEA})_{2} \mathrm{PbI}_{4}$ is also shown as dashed line for comparison(Data reproduced with permission from Ref. 38). (b) Theoretical spectrum of excitons obtained via DFT+GW+BSE simulations at $0 \mathrm{~K}$. The red and grey symbols represent the multiple bright and dark excitonic states identified in the theory, respectively. (c) DFT bandstructures for $(\mathrm{PEA})_{2} \mathrm{SnI}_{4}$ with electron and hole states (red areas) associated to three main exciton resonances identified in the theoretical optical spectrum as $X_{L E}, X_{H E-1}$ and $X_{H E-2}$.

A relatively broad yet clearly discernible resonance in the spectrum shown in Fig. 1 (a), centered around $2.3 \mathrm{eV}$ (labelled $X_{H E}$ ), suggests the presence of additional resonances within the continuum, which are not accounted for in the Elliott framework. Remarkably, $(\mathrm{PEA})_{2} \mathrm{PbI}_{4}$ does not exhibit features akin to $X_{H E}$ at higher energies despite the apparent structural similarity of both the materials. While this might indicate that the changes in the absorption spectrum due to the tin substitution may be the outcome of the reduced spin- 
orbit coupling, here we demonstrate, by using DFT and many body perturbation theory (MBPT) approaches, that the subtle structural variation of metal-halide layers arising from the organic-inorganic interactions holds the key.

Starting from experimental X-ray data ${ }^{7,41}$ we perform a set of full relaxation runs for several possible atomic structures, to identify the most stable geometries (Figs.S1,S2), ground state total energies and corresponding DFT bandstructures (Fig.S4). The energetically most stable structure, shown in Fig.2(a) (see also Fig. S1 in the SI), is governed by the positioning of the terminal amino group of the organic cation which determines the lattice configuration within the inorganic layer. We observe that the stable structure hosts two distinct tethering positions of the amine group with the inorganic lattice. This indirectly leads to two distinct yet stable octahedral configurations within the Sn-I network that are equally probable, shown in Fig.2(a) as differently colored structures. While we present these subtle structural variations within two neighbouring $2 \mathrm{D}$ layers for the sake of clarity, they can co-exist even within the same layer owing to the mutual independence of the organic cations. Accessibility of two equi-energetic lattice configurations created by the organic cation has been identified previously in 2D MHPs. ${ }^{42}$ Due to the random fluctuations in the position of the organic tethering, we consider that such distinct configurations are hard, if not impossible to be resolved in a typical X-ray scattering experiment. ${ }^{41}$

Using the most energetically stable structure, we include the role of electronic many-body effects both on the electronic and on the optical properties to allow a more quantitative comparison with the experimental steady state absorption spectrum. Due to low-dielectric screening and large quantum confinement, a wide renormalization of the DFT electronic gaps occurs and strongly bound excitons determine the optical properties of these layered perovskites. Similar to $(\mathrm{BA})_{2} \mathrm{PbI}_{4}$ reported by Palummo et al, ${ }^{43}$ the self-energy corrections open the DFT gap of about $1.5 \mathrm{eV}$ and produce a small reduction of the hole effective masses.

The quasi-particle band structure, obtained by using a self-consistent eigenvalue GW (ev-GW) approach, ${ }^{43-45}$ is shown in Fig.S3. The lowest four unoccupied conduction bands 


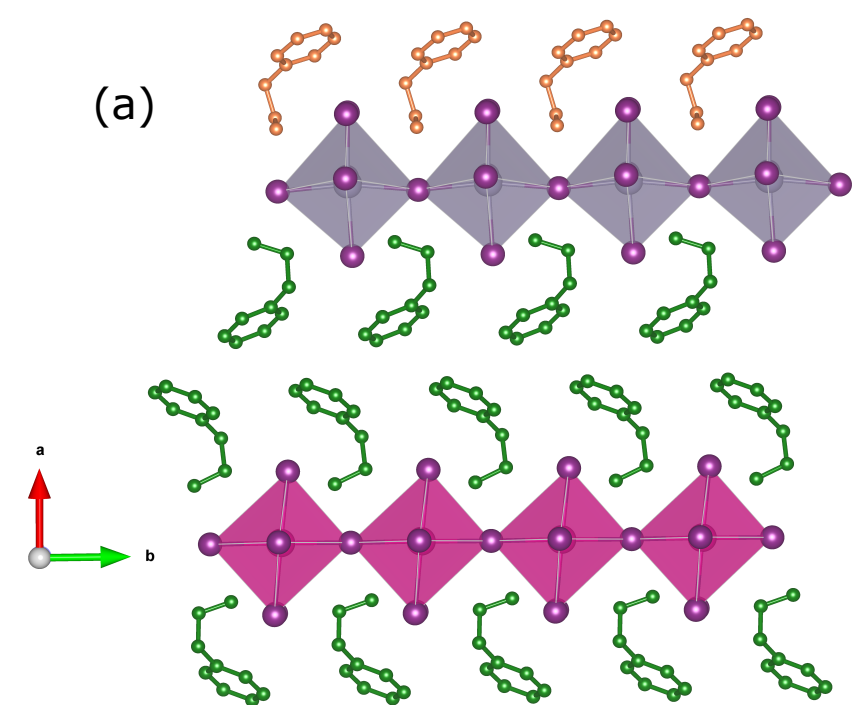

(b) $X_{\mathrm{LE}}$

(c) $\mathrm{X}_{\mathrm{HE}-1}$
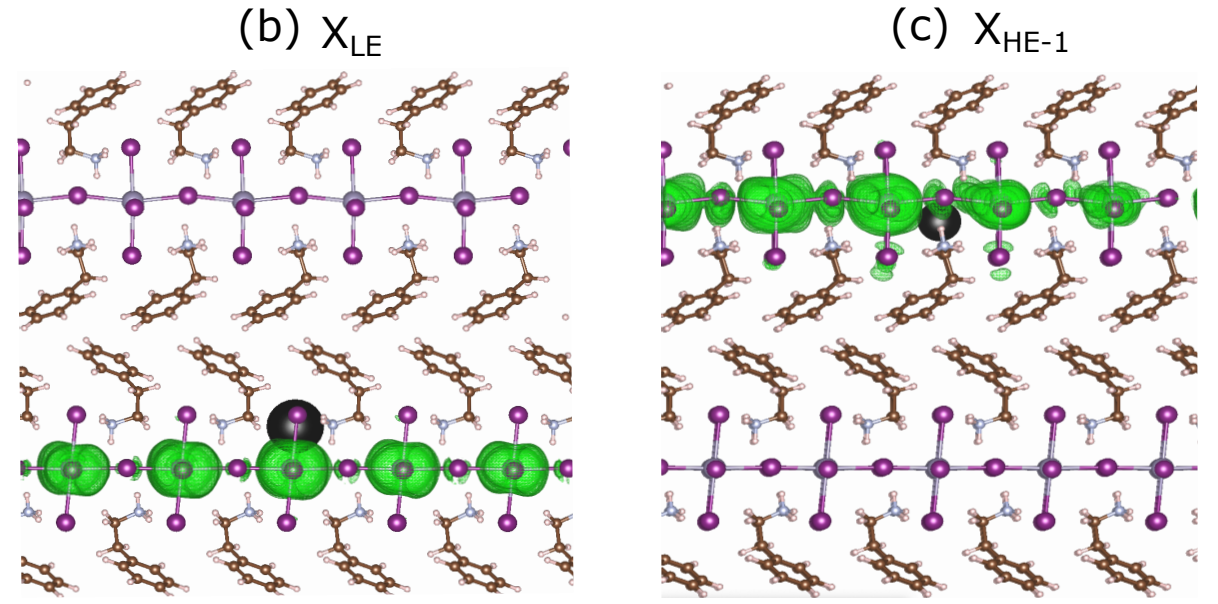

Figure 2: (a) Lateral view of the optimized most stable structure (1). For the sake of clarity $\mathrm{H}$ atoms are not indicated. Mauve and light purple octahedra indicate the non-equivalent geometrical rearrangement of the two inorganic layers after the full optimization. Green (orange) atoms are PEA cations with terminal in-plane (out-of-plane) C-N bonds [Mauve and light purple: Sn; Dark purple: I; Green and orange: PEA cations. Lateral view of (b) $X_{L E}$ and (c) $X_{H E-1}$ exciton wavefunction square modulus. The green isosurface is the probability of finding the electron when the hole position (gray large sphere) is fixed near a given atomic site.(Mauve: Sn, purple: I, brown: $\mathrm{C}$, light blue: $\mathrm{N}$, white: $\mathrm{H}$ atoms)

are separated by higher states by $100 \mathrm{meV}$ due to spin-orbit interaction (SOI), as shown by a comparison of DFT band structures with and without SOI contributions (Fig.S4). The analysis of the projected density of states (pDOS, Fig.S5) reveals that these unoccupied states are mainly due to the $p$ metal cation orbitals in the plane of the inorganic layer $\left(p_{y, z}\right)$. On the other hand, the four highest valence bands are associated to $p$ iodine orbitals with 
a non negligible contribution from $s$ states of tin. We also observe a splitting of $500 \mathrm{meV}$ between the top valence bands and as we discuss in the SI, this splitting originates from the slightly different lattice distortions of the two inorganic layers, mainly induced by the organic cation.

A direct comparison can now be made between the steady-state experimental and theoretical absorption spectra, shown in Fig. 1(c). It is worth to note that they are in good qualitative agreement: small remaining discrepancies in the position of excitonic peaks can be attributed to the lack of phonon interaction in the theoretical spectra, and to an incomplete convergence of higher energy structures due to limited number of transitions and k-points in the BSE solution (see Fig. S6).

The origin of the first experimental peak $X_{L E}$ is, as expected, excitonic in nature and, as shown in the left panel of Fig. 1(c), it is due to transitions from the two highest occupied to the four lowest unoccupied states near the A-point of the BZ. We consider that the broader peak at $X_{H E}$ has two main contributions, which we identified as $X_{H E-1}$ and $X_{H E-2}$ in Fig. 1(b). $X_{H E-2}$ involves the same transitions as $X_{L E}$, but occurring at other k-points near A and thus belongs to the same excitonic series. Instead, $X_{H E-1}$ involves transitions from two lower-lying valence bands (Fig.1(c), central panel), then being the first bright exciton of a new excitonic series similar to what is observed in 2D transition metal dichalcogenides (TMDs). ${ }^{46}$ However, contrary to the case of TMDs, the splitting here is of a completely different origin and as discussed previously, is a consequence of the organic-inorganic interactions.

Inspection of the square modulus of the exciton wavefunctions of $X_{L E}$ and $X_{H E-1}$ in the real-space gives further insights into the critical role of he lattice. Fig.2(b)-(c) show the conditional probability of locating the bound electron (green contour map) with the hole fixed in a probable position (black dot). It can be observed that the excitonic wavefunctions of $X_{L E}$ and $X_{H E-1}$ are localized within layers of distinct atomic distortions stabilized by different amine configurations.

The presence of the higher energy exciton series arising from multiple electronic bands is 
not only relevant for the linear absorption lineshape, but also can have non-trivial influence on carrier dynamics. Additional relaxation pathways beyond those which are phonon-driven may be present for the hot carriers, and may impede an efficient formation of the lightemitting lower energy excitons. Accordingly, we investigate the photo-excitation dynamics in $(\mathrm{PEA})_{2} \mathrm{SnI}_{4}$ and their temperature dependence using ultrafast differential transmission (DT) spectroscopy.

We acquire the DT spectra - time and spectrally resolved changes in the transmission of a probe pulse induced by a pump pulse - with the pump energy tuned to the energy of $X_{L E}$ at $1.96 \mathrm{eV}$, thus resonantly generating the low energy excitons, and with the pump energy at $3.1 \mathrm{eV}$ associated to hot carrier excitation. In Fig. 3 we show the normalized DT spectra at different pump-probe delays for resonant ((a), (b)) and hot carrier ((c), (d)) excitations. We highlight the spectral dynamics at two different spectral regions around the energies of $X_{L E}$ and $X_{H E}$ in the linear absorption spectrum, also shown along with with DT spectra. All the spectra in Fig. 1 are acquired at $77 \mathrm{~K}$ and on the same sample.

It is clear the DT dynamics are defined by the presence of multiple time-evolving and overlapping bands. For the sake of convenience, we label the relevant positive photo-bleach (PB) bands with $\Gamma$, stimulated emission (SE) bands with $\Phi$ and negative photo-induced absorption (PA) bands with $\Pi$. The primary spectral feature in both the excitation conditions is a prominent positive $\Delta T / T$ signal at $1.95 \mathrm{eV}$ labelled as $\Gamma_{1}$, at the edge of $X_{L E}$. We also observe a positive $\Delta T / T$ shoulder on the blue side of $\Gamma_{1}$, labelled as $\Gamma_{1}^{*}$. We consider both $\Gamma_{1}$ and $\Gamma_{1}^{*}$ to be signatures of photo-bleach of a subset of excitonic transitions at $X_{L E}$ where multiple bright and dark excitonic resonances are predicted by our calculations (Fig. 1.(b)). We note that $\Gamma_{1}$ may have an additional contribution from SE. On the red-end of $\Gamma_{1}$ there are two PA bands, $\Pi_{1}$ and $\Pi_{1}^{*}$ and another positive $\Delta T / T$, labelled $\Phi$. Due to the absence of any feature in the linear spectra and to the presence of a photoluminescence (PL) band at the energy of $\Phi$, we assign it to SE.

We also observe a broad featureless negative band extending from $1.97 \mathrm{eV}$ to $2.2 \mathrm{eV}$, which 

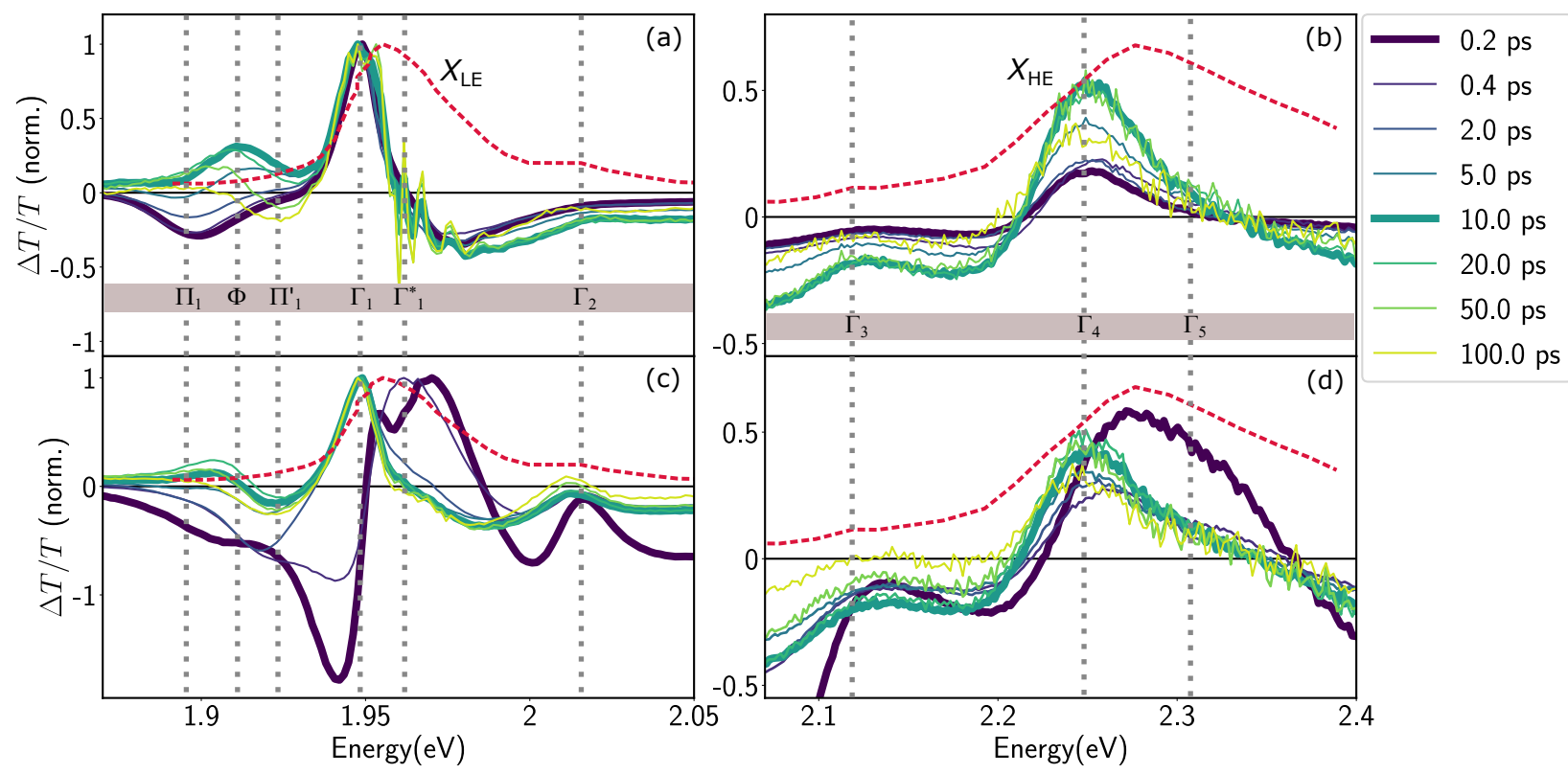

Figure 3: Differential transmission spectra of (PEA) ${ }_{2} \mathrm{SnI}_{4}$ upon (a),(b) resonant excitation $\left(E_{\text {pump }}=1.96 \mathrm{eV}\right)$ of the lowest lying excitonic states $(\mathrm{c}),(\mathrm{d})$ non-resonant excitation of hot free carriers $\left(E_{\text {pump }}=3.1 \mathrm{eV}\right)$. The DT signal both in the (a),(c) $X_{L E}$ and (b),(d) $X_{H E}$ regions is normalized to the maximum in $X_{L E}$. As a reference, the steady-state absorbance spectrum is shown as a red dashed line.

is typically associated to the photo-induced changes in the refractive index across the entire spectrum. ${ }^{47}$ Atop this broad negative feature, we may identify two "positive-going" features at $2.02 \mathrm{eV}$ and $2.13 \mathrm{eV}$, more prominent for the high energy excitation, with associated low oscillator strength resonances in the linear spectrum: accordingly, we identify overlapping PB bands $\Gamma_{2}$ and $\Gamma_{3}$ at those energies. Additional PB bands $\Gamma_{4}$ and $\Gamma_{5}$ are also seen at $X_{H E}$, with the latter being a low intensity shoulder to $\Gamma_{4}$ and more prominent with $E_{\text {pump }}=3.1 \mathrm{eV}$.

Upon resonant excitation at $1.93 \mathrm{eV}$, we observe an instantaneous appearance of $\Gamma_{1}+$ $\Gamma_{1}^{*}$ bands owing to exciton generation within the excitation pulse duration. This is also supported by a well defined $\Pi_{1}$ feature associated to the exciton $\rightarrow$ biexciton transition. ${ }^{48} \mathrm{~A}$ lower lying exciton state is populated in a few picoseconds resulting in a SE feature at $\Phi$. Similar inter-exciton dynamics have been observed both in $(\mathrm{PEA})_{2} \mathrm{PbI}_{4}$ by Thouin et al ${ }^{49}$ and in $(\mathrm{PEA})_{2} \mathrm{SnI}_{4}$ by Folpini et al. ${ }^{21}$ The details of the dynamics in this spectral range are hard to isolate due to the close spectral overlap between different features: in particular, 
the temporal evolution of negative features $\Pi_{1}$ and $\Pi_{1}^{*}$, both of which can associated to the exciton PA, is partially obscured by the competing growth in the intensity of positive features such as $\Phi$. Such a spectral congestion can be addressed by using two-dimensional spectroscopy,${ }^{6,48}$ which is out of the scope of the current work.

In the $\mathrm{X}_{H E}$ range of the DT spectrum, shown in Fig 3(b), we observe the instantaneous appearance of a small positive going feature at $\Gamma_{3}$ and a clear PB feature at $\Gamma_{4}$, which matches with the lower energy portion of the $X_{H E}$ in the linear absorption spectrum. Based on the theoretical calculations presented earlier, we assign $\Gamma_{4}$ to the PB of states similar to $X_{H E-1}$ (see Fig. 1(b)), associated to the higher lying excitonic states made up of carriers from the lower lying VB, and localized within the metal-halide layers which are coordinated by mutually perpendicular amine groups (Fig. 2(b)). Thus, here we are observing the PB of $X_{H E-1}$ even though we are resonantly generating $X_{L E}$ excitons, even though they share neither a common ground state in the excitonic phase space, nor the relevant orbitals in the real-space. We interpret this as a consequence of phase-space filling of electronic states in the conduction band states that are common to both $X_{L E}$ and $X_{H E-1}$, see Fig. 1(c) (left and center panels). Even though $X_{H E-1}$ states are not populated under this condition, presence of $X_{L E}$ population reduces the available density of states in the $\mathrm{CB}$, thus reducing the oscillator strength of $X_{H E-1}$ resulting in $\Gamma_{4}$. The relative intensity of $\Gamma_{4}$ to $\Gamma_{1}$ increases at longer delays: this is a consequence of the transfer of population within the exciton finestructure at $X_{L E}$ and possibly to the lower lying dark states, predicted around $1.98 \mathrm{eV}$ and $2 \mathrm{eV}$ by our theoretical analysis.

Despite the complex spectral structure, a simple photophysical scenario may be inferred from Fig.3: resonant excitation generates exciton $\left(X_{L E}\right)$ population with an associated PB (and possibly SE) at $\Gamma_{1}$ and $\Gamma_{1}^{*}$, PA at $\Pi_{1}$ and $\mathrm{PB}$ at $\Gamma_{4}$ due to the phase-space filling of the $\mathrm{CB}$ states that contribute to the composition of $X_{H E-1}$ exciton. In a few picoseconds, population is transferred from $\Gamma_{1}$ to a lower lying emissive exciton state with $\mathrm{SE}$ at $\Phi$. Overall the exciton population relaxes to the ground state in hundreds of picoseconds, as 
also substantiated by the PL dynamics discussed in 21 .

Generation of hot carriers with a pump energy much higher than that of the $X_{L E}$ exciton (at $3.1 \mathrm{eV}$ ) presents a different dynamic scenario (Figs. 3(c)-(d)). Firstly, $\Gamma_{1}$ and $\Gamma_{1}^{*}$ appear in a couple of picoseconds and not instantaneously. At early times, there is instead a derivativelike lineshape $d \Gamma_{1}^{*}$ at their energy, indicative of a red-shift in the exciton $\left(X_{L E}\right)$ energy. The absence of the derivative feature in the resonant excitation indicates its origin to be the hot charge carriers which screen the Coulomb interactions of the exciton, resulting in a bandgap renormalization (BGR) and a red-shift of the $X_{L E}$ exciton absorption feature. In a few picoseconds, this lineshape evolves into the features observed in the resonant excitation case which include $\Gamma_{1}, \Gamma_{1}^{*}$ and $\Phi$. The absence of the SE feature, $\Phi$, in the presence of hot carriers in the early times is a result of the dominant contribution of negative features (from the derivative lineshapes), which are spectrally overlapped.

We observe correlated picosecond spectral dynamics also in the $X_{H E}$ energy range, especially in the dynamics of the PB feature $\Gamma_{5}$ whose energy matches with that of $X_{H E-2}$ states: these higher energy excitonic resonances are composed of the hot carrier states in the electronic bands shown in Fig. 1(c), right panel. Accordingly, we assign $\Gamma_{5}$ to the population in $X_{H E-2}$ and we observe that it rapidly decays along with the derivative feature at $\Gamma_{1}$. This results in an apparent red-shift of the overall $\mathrm{PB}$ feature at $X_{H E}$, that finally settles at $\Gamma_{4}$ much alike the resonant case. We interpret the correlated growth dynamics at $\Gamma_{1}(1.95 \mathrm{eV})$ and red-shift towards $\Gamma_{4}(2.25 \mathrm{eV})$ as signatures of hot carrier thermalization and exciton formation that happen over a couple of picoseconds at $77 \mathrm{~K}$.

\section{Discussion}

Spectral congestion with overlapping features in the spectra shown Fig. 3(c) prevents a robust analysis of the spectral dynamics at $X_{L E}$, even for DT spectra taken at $5 \mathrm{~K}$ (see Fig.S8). At higher temperatures, this task is even more challenging due to the temperature induced 
broadening of the overlapping spectral features. Nevertheless, we construct an empirical yet quantitative model that can capture the most salient characteristics of the observed DT dynamics. We consider that the DT spectrum in the $X_{L E}$ region $(1.88-1.96 \mathrm{eV})$ at $500 \mathrm{fs}$ and at $5 \mathrm{~K}$ is composed of four components: (i) a PA band (П) signature centered around $1.89 \mathrm{eV}$ (ii) a relatively narrow positive $\Delta T / T$ band centered at $1.915 \mathrm{eV}\left(\Gamma_{1}^{*}\right)$, (iii) a slightly broader peak at $1.935 \mathrm{eV}\left(\Gamma_{1}\right)$ and (iv) a first derivative of the peak at $1.935 \mathrm{eV}(d \Gamma)$. Considering Gaussian spectral lineshapes, we reproduce the DT spectra at all the pump-probe delays with time dependent amplitudes of the four associated components. The fitting procedure, described in more detail in SI yields robust and accurate fits from $5 \mathrm{~K}$ up to $300 \mathrm{~K}$; a comparison of experimental data and fit at $5 \mathrm{~K}$ shown in Fig. S10.

The peak positions of $\Gamma_{1}^{*}, \Gamma_{1}$ and $\Pi_{1}$ (Fig.S11(a)) show a consistent blue shift with increasing temperature and a constant energy separation of $40 \mathrm{meV}$ between them: this corroborates the assignment of $\Pi_{1}$ as PA to a biexciton with $40 \mathrm{meV}$ binding energy, in line with previously reported values. ${ }^{48}$ Their respective linewidths are also comparable, although the linewidth of $\Gamma_{1}$ is consistently smaller at all temperatures, see Fig. 4(b). The increasing linewidth with temperature is clearly an outcome of increased scattering with phonons. We fit the linewidth $\Delta E_{\Gamma_{1}^{*}}$ as a function of temperature with the dispersion rela$\operatorname{tion}^{50} \Delta E_{\Gamma_{1}^{*}}(T)=G+\gamma /\left(e^{E_{L O} / k_{B} T}-1\right)$, where $G$ represents the temperature independent inhomogeneous broadening and $k_{B}$ is the Boltzmann constant: we obtain the exciton-phonon coupling constant $\gamma$ of $10 \mathrm{meV}$, with the LO phonon energy $E_{L O}$ estimated to be $15 \mathrm{meV}$. Strikingly, these numbers are comparable to the phonon energy of $17 \mathrm{meV}$ estimated for $(\mathrm{PEA})_{2} \mathrm{PbI}_{4}$ by Neutzner et al. ${ }^{38}$ This suggests that the vibrational landscape and the nature of exciton-phonon interactions in (PEA)SnI 4 is not very different from that of the lead counterpart, apart from a minor modification in the phonon energy owing to the lighter metal ion. We highlight however that this estimate of the exciton-phonon coupling can only be considered qualitative and a rigorous analysis of coherent nonlinear lineshapes is essential to extract experimentally accurate parameters as discussed by Thouin et al.$^{51}$ 

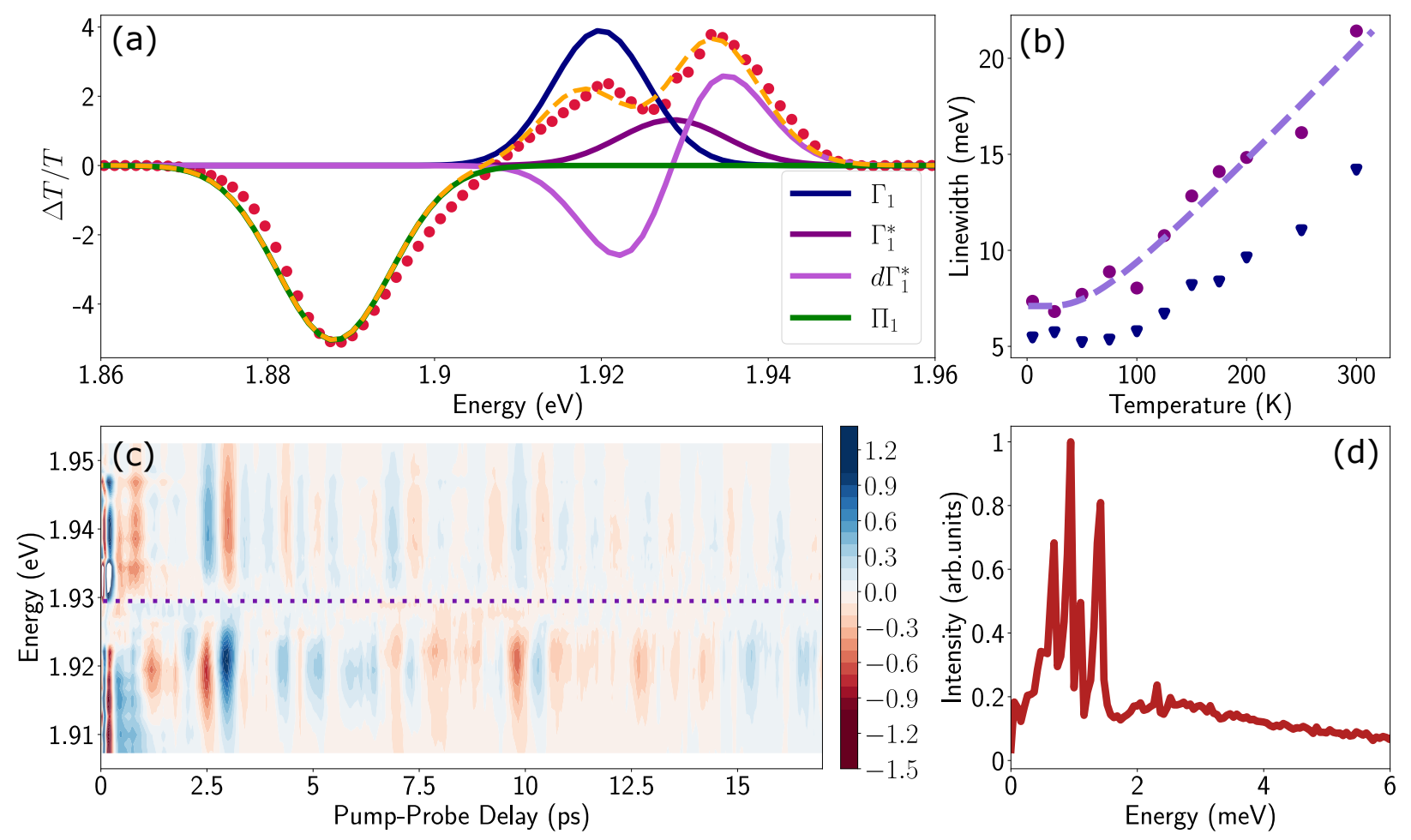

Figure 4: a) Differential transmission spectrum, for $\mathrm{T}=5 \mathrm{~K} \tau=0.5 \mathrm{ps}$ (red dots) and corresponding lineshape fit (dashed line). Schematic fit model: the fit model involves the $\mathrm{PB}$ of $\Gamma_{1}$ and $\Gamma_{1}^{*}$ (positive gaussian features), the energy shift of the excitonic state (derivative gaussian shape $d \Gamma_{1}^{*}$ ) and corresponding $\mathrm{PA} \Pi_{1}$ (negative gaussian feature). b) Widths of the fit components for $\Gamma_{1}$ (triangles) and $\Gamma_{1}^{*}$ (circles). A fit with the dispersion relation of optical phonons (dashed red line) gives an estimate of the LO phonon energy of $14.7 \mathrm{meV}$. c) Two dimensional map showing the time resolved coherent oscillations upon hot carrier excitation pumping at $3.1 \mathrm{eV}$, as a function of pump delay time and detection energy. The observed oscillations persist for longer than $20 \mathrm{ps}$. The nodal point at $\mathrm{E}=1.927 \mathrm{eV}$ marks the position of the exciton coupled to the coherent phonons. d) Fourier transform spectrum of the oscillatory dynamics displayed in (c), and integrated over all the probe energies. This corresponds to the resonant impulsive stimulated Raman spectrum of $(\mathrm{PEA})_{2} \mathrm{SnI}_{4}$ at $5 \mathrm{~K}$.

We can verify this more rigorously by analyzing the coherent oscillations in the $\Delta T / T$ response at $5 \mathrm{~K}$. The ultrashort pump pulse impulsively generates a coherent vibrational wavepacket through resonant impulsive stimulated scattering (RISRS). ${ }^{7}$ The evolution of the wavepacket along the potential energy surface of the ground state results in an oscillatory DT signal superimposed on the independently evolving dynamics at the energy of $X_{L E}$. Given the large anharmonicity of the 2D MHP lattice, these oscillations are typically visible only at low temperatures where the phonon-phonon scattering is minimum, thus explaining their 
dominant role at $5 \mathrm{~K}$. The coherent oscillations, obtained by subtracting the decay dynamics (modelled as a $5^{\text {th }}$ order polynomial), are shown as a 2D beating map in Fig 4(c): their Fourier transform along the pump-probe delay integrated over probe energies (Fig. 4 (d)) represents the spectrum of optical phonons generated via the RISRS process. We observe multiple peaks below $2 \mathrm{meV}$, with a broad tail extending up to $10 \mathrm{meV}$. The main components have a frequency $\nu=0.2-0.4 \mathrm{THz}$, suggesting a dominant contribution from the motion of the Sn-I octahedra, much like in( $\mathrm{PEA})_{2} \mathrm{PbI}_{4}$. Intriguingly despite the lighter tin ion the observed energies are slightly lower than the lead counterpart. 7,52

The coherent phonons generated in the RISRS experiment represent the lattice modes that are coupled to the electronic transitions resonant with the pump energy. Thus, the modes shown in Fig. 4(d) upon excitation at $3.1 \mathrm{eV}$, are those coupled to free carriers. The reason to observe them at the energy of $X_{L E}$ is that their evolution in the ground state also modulates the absorption of the excitonic resonances they are dressing.

We observe a characteristic structure of the modulation amplitude, with a nodal null point at $1.927 \mathrm{eV}$ together with a $\pi$-shift in the relative phase of the oscillations at this probe energy. Such a nodal point represents the peak-energy of the resonance which is coupled to and modulated by the coherent phonons. ${ }^{6,7}$ It is worthwhile to note that this energy does not correspond to the peak energy of $X_{L E}$ in linear absorption, which suggests that $X_{L E}$ is composed of multiple broad resonances, only one of which is being dressed by these phonons. Coincidentally, the nodal point energy matches exactly with the peakposition of $\Gamma_{1}^{*}$ considered in the DT spectral analysis (see Fig. 4(b)). Notably, the resonance at $\Gamma_{1}$ is not coupled to the generated coherent phonons.

Two explanations may be invoked to rationalize this observation. One follows the work of Thouin et al, ${ }^{7}$ where $\Gamma_{1}$ and $\Gamma_{1}^{*}$ represent two exciton-polaron states that are distinctly dressed by the lattice phonons. Alternatively, we can consider that $\Gamma_{1}$ is a stimulated emission band, given that it coincides with one of the peaks in the PL spectrum (see fig.S11(a),S12). RISRS signal arises due to the evolution of the vibrational wavepacket in the electronic 
ground-state and thus the excited-state emission rate will not be modulated: thus, one expects to observe the RISRS exclusively at the bleach signatures that probe the absorption probabilities and not in the SE features. The relatively narrow linewidth of $\Gamma_{1}$ further corroborates this assignment.

We will now elaborate on the dynamics of the fit parameters, shown in Fig.S10,S11, hypothesizing that $\Gamma_{1}$ is indeed a SE signature. Any discrepancy in this assignment however will not drastically affect the derived photo-physical model, since SE as well as a PB may be used as spectral signatures for the evolving exciton population. Firstly, after a few picoseconds, the DT spectrum is composed of $\Gamma_{1}, \Gamma_{1}^{*}$ and $\Pi_{1}$, all of which show correlated decays over hundreds of picoseconds, consistent with the PL dynamics of the relaxation of the photo-excited charge carriers. ${ }^{21}$ Atop the decay, we also observe a growth in the intensity of $\Gamma_{1}^{*}$ within $250 \mathrm{ps}$, particularly evident at temperatures below $50 \mathrm{~K}$. We had previously discussed these dynamics in the context of population exchange between dark and bright excitonic states in Ref. ${ }^{21}$

At early times $(<10 \mathrm{ps})$, we observe the derivative component at $\Gamma_{1}^{*}\left(\mathrm{~d} \Gamma_{1}^{*}\right.$ in Fig. 4(a)) disappearing in about 3 ps at $5 \dot{\mathrm{K}}$ and less than $500 \mathrm{fs}$ at RT. This spectral feature indicates a photo-induced red-shift in the exciton $\left(\Gamma_{1}^{*}\right)$ energy, which is a consequence of screening of Coulomb interactions by hot charge-carriers and subsequent BGR. Notably, the screening of exciton absorption is perceived at the energy of $\Gamma_{1}^{*}$, which further substantiates our assignment of $\Gamma_{1}^{*}$ to $\mathrm{PB}$ and $\Gamma_{1}$ to SE. The screening and accordingly $d \Gamma_{1}^{*}$ is quenched as the hot carriers thermalize to form bound excitons over a few picoseconds. This timescale exactly matches with the formation dynamics of $\Gamma_{1}$, which is a finger print of phase-space filling of the excitonic state: accordingly this timescale represents ultrafast exciton formation that follows hot carrier thermalization. Similar timescales for exciton formation have been reported with more ambiguous $\mathrm{THz}$ probes by Folpini el al in $(\mathrm{BA})_{2} \mathrm{PbI}_{4} \cdot{ }^{53}$ 

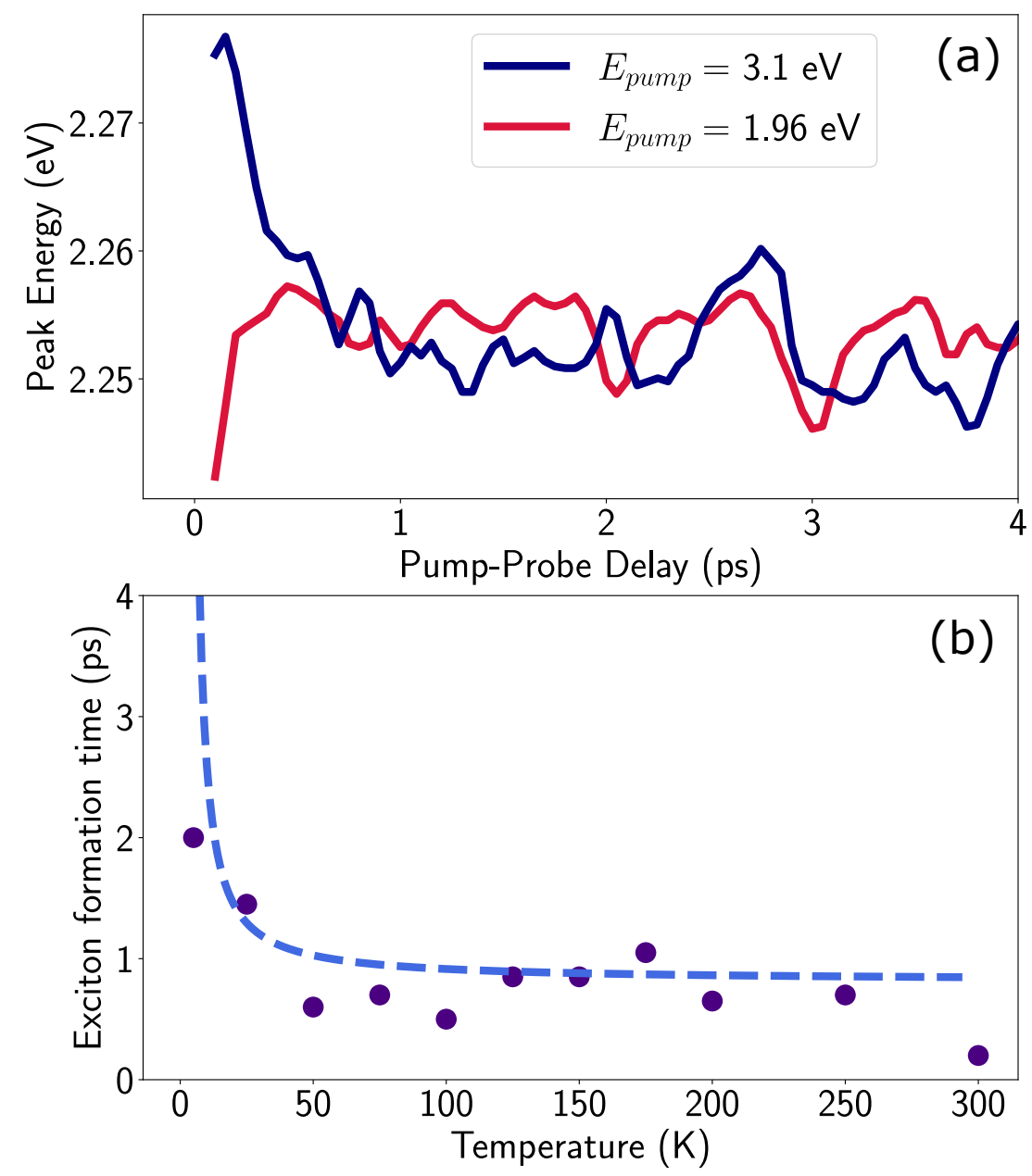

Figure 5: a) Peak position of the $X_{H E}$ feature at $\mathrm{T}=77 \mathrm{~K}$ as a function of pump-probe delay : while for excitation resonant to the excitonic transition (red) no spectral shift occurs, for excitation in the continuum (blue) a red shift occurs in the first picosecond after excitation b) Thermalization time as a function of temperature: as a figure of merit, we consider the time it takes for the $X_{H E}$ peak to match its equilibrium values at long delays when excited in the continuum, up to a $95 \%$ tolerance.

As noted earlier, the DT dynamics at $X_{H E}$ also are indicative of ultrafast hot carrier thermalization and exciton formation, spectral signature of which lies in the apparent redshift in the peak energy of the PB feature at $X_{H E}$. The dynamics of the corresponding peak-shift at $77 \mathrm{~K}$ are shown in Fig. 5(a) for the resonant and hot carrier excitation case 
(red and blue line respectively): while no red shift is observed in the resonant case, the hot carriers dynamics match those observed in $X_{L E}$, specifically in the loss of the derivative feature $d \Gamma_{1}^{*}$ and the appearance of $\Gamma_{1}$.

Given the correlated appearance of $\Gamma_{1}^{*}$, the red shift of $X_{H E}$ can be used as a figure of merit to compare exciton formation time across different temperatures. The effective thermalization time $\tau_{\text {therm }}$, defined as the delay when the peak position of $X_{H E}(\tau)$ is within $90 \%$ of its equilibrium value at long delays (1 ns), is shown in fig.5(b) (the full time dependent peak positions as a function of temperature are shown in fig.S9). This estimate gives an ultrafast exciton formation of less than 2 ps even at $5 \mathrm{~K}$, and points to a scenario in which a large fraction of the excited carriers rapidly relax to the bottom of the band structure due to effective electron-phonon coupling and form stable exciton population. The temperature dependence of the formation time is consistent with exciton thermalization driven by scattering of the carriers with LO phonons, ${ }^{54,55}$ assuming the phonon energy to be $0.5 \mathrm{meV}$ (4 $\mathrm{cm}^{-1}$ ) as estimated from the RISRS analysis (see Fig. 4(d)).

Despite the ultrafast thermalization dynamics and clear spectroscopic evidence of exciton formation, we highlight that even after hundreds of picoseconds the DT spectra obtained with the pump at $3.1 \mathrm{eV}$ do not resemble the resonant excitation spectra. This is particularly striking at the $X_{L E}$ spectral range as highlighted in Fig. 6 where we compare the normalized DT spectra under the two pump conditions at $5 \mathrm{ps}$ and $250 \mathrm{ps}$. Two pertinent differences can be identified: (a) the absence of the SE feature, $\Phi(1.92 \mathrm{eV})$ that we had attributed to the population of the lower lying excitonic state with the hot carrier excitations and (b) the absence of $\Gamma_{2}(2.01 \mathrm{eV})$ bleach feature with the resonant excitation. We also note that in the $X_{H E}$ region the $\Gamma_{5}$ feature has not completely disappeared even after hundreds of picoseconds. The sustained bleach signatures at $\Gamma_{2}$ and $\Gamma_{5}$ can be interpreted as the statefilling of higher lying excitonic states that trap a part of the photo-generated hot carrier population. Whilst $\Gamma_{5}$ feature can be attributed to the $X_{H E-2}$ excitonic state predicted by the BSE calculations, $\Gamma_{2}$ (and $\Gamma_{3}$ ) is not theoretically predicted. 

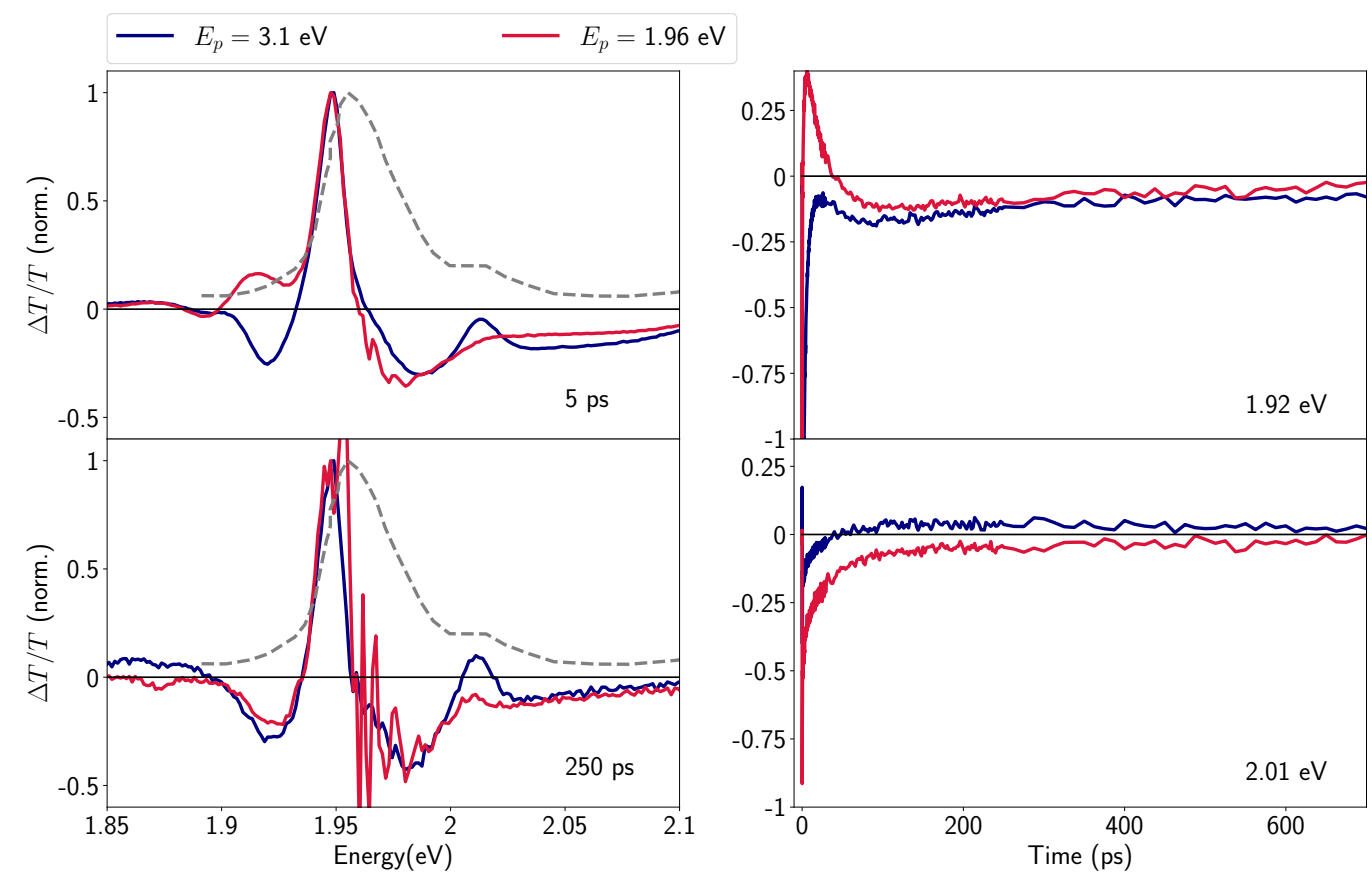

Figure 6: Differential transmission spectrum of the $\mathrm{X}_{L E}$ range at a) 5 ps c) 250 ps delay, showing long lived thermalization dynamics: differences between resonant (red line) and nonresonant (blue line) excitation are still found after hundreds of picoseconds. b),d) Dynamics at the spectral position of b) $\Pi_{1}$ and d) $\Gamma_{2}$ respectively on the red and blue side of the resonant pump, showing contributions of opposite sign for tens $\left(\Pi_{1}\right)$ and hundreds of ps $\left(\Gamma_{2}\right)$.

\section{Conclusions}

We have investigated the effect of the metal cation substitution on the optical properties of a 2D MHP. Comparing the static absorbance of $(\mathrm{PEA})_{2} \mathrm{PbI}_{4}$ and $(\mathrm{PEA})_{2} \mathrm{SnI}_{4}$ reveals the appearance of higher energy resonances upon substitution of lead with tin. Based on DFT and MBPT calculations, we identify multiple excitonic resonances in the probed spectral range which are composed of excitations at various regions of the carrier phase-space. Importantly, we predict the presence of two valence bands with a splitting energy of about $500 \mathrm{meV}$ that result in two distinct excitonic series - $X_{L E}$ and $X_{H E-1}$. Even though they originate from holes from different valence bands, they share the same electron bands in the conduction band. This leads to the detection of PB signatures of $X_{H E-1}$ in differential 
transmission spectroscopy, even when $X_{L E}$ states are uniquely excited. We also investigated the ultrafast hot carrier thermalization dynamics and exciton formation dynamics and their temperature dependence to evaluate the role of exciton-phonon interactions. Notably, while we observe a change in the energy of the phonons due to the tin substitution, the nature of exciton-phonon interactions remains unaffected by the choice of the metal cation. In addition to the picosecond phonon-assisted thermalization, the presence of $X_{H E-1}$ and other higher energy excitonic states result in a slower relaxation channel for the photo-excited population towards the lowest energy emissive exciton at $X_{L E}$. Such an excitonic landscape offers a possibility to create high energy reservoirs of exciton population that preserve the photoexcited population from many-body interactions and other loss channels prevalent in high density applications, particularly in lasers. On a more fundamental level, the splitting of the valence band and resultant co-existence of multiple excitonic series suggested in this work originate from the subtle modulations imparted by the organic-inorganic interactions on the inorganic component of the lattice. This highlights the importance and need for a rigorous evaluation of the structural complexity and dynamics of 2D MHPs, and their relevance in the excitonic structure.

\section{Acknowledgements}

MP and GG acknowledge for the availability of high-performance computing resources at Cineca under Iscra-B and iscra-C initiatives" M.P. acknowledges the financial support of INFN Time2quest project.

\section{Author Contributions}

The experimental measurements were performed by GF and LM under the supervision of GC and ARSK. The data analysis was performed by GF and ARSK. The sample was fabricated by DC under the supervision of AP. The theoretical calculations and analysis were performed 
by MP and GG. The manuscript was prepared by GF, MP, GG and ARSK with inputs from all the authors. The project was conceived by ARSK. ARSK.

\section{References}

\section{References}

1. Kagan, C. R.; Mitzi, D. B.; Dimitrakopoulos, C. D. Organic-inorganic hybrid materials as semiconducting channels in thin- film field-effect transistors. Science 1999, 286, 945947.

2. Knutson, J. L.; Martin, J. D.; Mitzi, D. B. Tuning the band gap in hybrid tin iodide perovskite semiconductors using structural templating. Inorganic Chemistry 2005, 44, 4699-4705.

3. Shimizu, M.; Fujisawa, J. I.; Ishi-Hayase, J. Influence of dielectric confinement on excitonic nonlinearity in inorganic-organic layered semiconductors. Physical Review B Condensed Matter and Materials Physics 2005, 71, 1-9.

4. Chen, Y.; Sun, Y.; Peng, J.; Tang, J.; Zheng, K.; Liang, Z. 2D Ruddlesden-Popper Perovskites for Optoelectronics. Advanced Materials 2018, 30, 1-15.

5. Liu, X. K.; Xu, W.; Bai, S.; Jin, Y.; Wang, J.; Friend, R. H.; Gao, F. Metal halide perovskites for light-emitting diodes. Nature Materials 2020,

6. Srimath Kandada, A. R.; Silva, C. Exciton Polarons in Two-Dimensional Hybrid MetalHalide Perovskites. The Journal of Physical Chemistry Letters 2020, 11, 3173-3184.

7. Thouin, F.; Valverde-Chávez, D. A.; Quarti, C.; Cortecchia, D.; Bargigia, I.; Beljonne, D.; Petrozza, A.; Silva, C.; Srimath Kandada, A. R. Phonon coherences reveal 
the polaronic character of excitons in two-dimensional lead halide perovskites. Nature materials 2019, 18, 349-356.

8. Rusydi, A.; Chi, X.; Leng, K.; Li, R.; Yu, X.; Yang, P.; Breese, M.; Loh, K. P. Observation of strong helical excitons and unexpectedly giant Rashba splitting in single crystal twodimensional Organic-Inorganic Halide Perovskites. 2020,

9. Tao, W.; Zhou, Q.; Zhu, H. Dynamic polaronic screening for anomalous exciton spin relaxation in two-dimensional lead halide perovskites. Science advances 2020, 6, 2-10.

10. Ishihara, T.; Hong, X.; Ding, J.; Nurmikko, A. V. Dielectric confinement effect for exciton and biexciton states in PbI4-based two-dimensional semiconductor structures. Surface Science 1992, 267, 323-326.

11. Goto, T.; Makino, H.; Yao, T.; Chia, C. H.; Makino, T.; Segawa, Y.; Mousdis, G. A.; Papavassiliou, G. C. Localization of triplet excitons and biexcitons in the two-dimensional semiconductor (C H3 C6 H4 C H2 N H3) 2 Pb Br4. Physical Review B - Condensed Matter and Materials Physics 2006, 73, 1-5.

12. Blancon, J. C.; Even, J.; Stoumpos, C. C.; Kanatzidis, M. G.; Mohite, A. D. Semiconductor physics of organic-inorganic 2D halide perovskites. Nature Nanotechnology 2020, 15, 969-985.

13. Cortecchia, D.; Neutzner, S.; Kandada, A. R. S.; Mosconi, E.; Meggiolaro, D.; De Angelis, F.; Soci, C.; Petrozza, A. Broadband emission in two-dimensional hybrid perovskites: The role of structural deformation. Journal of the American Chemical Society 2017, 139, $39-42$.

14. Cortecchia, D.; Yin, J.; Petrozza, A.; Soci, C. White light emission in low-dimensional perovskites. Journal of Materials Chemistry C 2019, 7, 4956-4969. 
15. Febriansyah, B.; Borzda, T.; Cortecchia, D.; Neutzner, S.; Folpini, G.; Koh, T. M.; Li, Y.; Mathews, N.; Petrozza, A.; England, J. Metal coordination sphere deformation induced highly Stokes-shifted, ultra broadband emission in 2D hybrid lead-bromide perovskites and investigation of its origin. Angewandte Chemie International Edition 2020,

16. Yuan, M.; Quan, L. N.; Comin, R.; Walters, G.; Sabatini, R.; Voznyy, O.; Hoogland, S.; Zhao, Y.; Beauregard, E. M.; Kanjanaboos, P., et al. Perovskite energy funnels for efficient light-emitting diodes. Nature nanotechnology 2016, 11, 872-877.

17. Yusoff, A. R. b. M.; Nazeeruddin, M. K. Low-Dimensional Perovskites: From Synthesis to Stability in Perovskite Solar Cells. Advanced Energy Materials 2018, 8, 1702073.

18. Yantara, N.; Bruno, A.; Iqbal, A.; Jamaludin, N. F.; Soci, C.; Mhaisalkar, S.; Mathews, N. Designing Efficient Energy Funneling Kinetics in Ruddlesden-Popper Perovskites for High-Performance Light-Emitting Diodes. Advanced Materials 2018, 30, 1-7.

19. Qin, C.; Sandanayaka, A. S.; Zhao, C.; Matsushima, T.; Zhang, D.; Fujihara, T.; Adachi, C. Stable room-temperature continuous-wave lasing in quasi-2D perovskite films. Nature 2020, 585, 53-57.

20. Ishihara, T.; Takahashi, J.; Goto, T. Optical properties due to electronic transitions in two-dimensional semiconductors (CnH2n+1NH3)2PbI4. Physical Review B 1990,

21. Folpini, G.; Cortecchia, D.; Petrozza, A.; Srimath Kandada, A. R. The role of dark exciton reservoir in the luminescence efficiency of two-dimensional tin halide perovskites. Journal of Materials Chemistry C 2020,

22. Liang, H.; Yuan, F.; Johnston, A.; Gao, C.; Choubisa, H.; Gao, Y.; Wang, Y. K.; Sagar, L. K.; Sun, B.; Li, P.; Bappi, G.; Chen, B.; Li, J.; Wang, Y.; Dong, Y.; Ma, D.; Gao, Y.; Liu, Y.; Yuan, M.; Saidaminov, M. I. et al. High Color Purity Lead-Free Perovskite Light-Emitting Diodes via Sn Stabilization. Advanced Science 2020, 7. 
23. Lanzetta, L.; Marin-Beloqui, J. M.; Sanchez-Molina, I.; Ding, D.; Haque, S. A. TwoDimensional Organic Tin Halide Perovskites with Tunable Visible Emission and Their Use in Light-Emitting Devices. ACS Energy Letters 2017, 2, 1662-1668.

24. Xu, H.; Jiang, Y.; He, T.; Li, S.; Wang, H.; Chen, Y.; Yuan, M.; Chen, J. Orientation Regulation of Tin-Based Reduced-Dimensional Perovskites for Highly Efficient and Stable Photovoltaics. Advanced Functional Materials 2019, 29, 1-11.

25. Cao, D. H.; Stoumpos, C. C.; Yokoyama, T.; Logsdon, J. L.; Song, T. B.; Farha, O. K.; Wasielewski, M. R.; Hupp, J. T.; Kanatzidis, M. G. Thin Films and Solar Cells Based on Semiconducting Two-Dimensional Ruddlesden-Popper (CH3(CH2)3NH3)2(CH3NH3)n1SnnI3n+1 Perovskites. ACS Energy Letters 2017, 2, 982-990.

26. Zhang, X.; Wang, C.; Zhang, Y.; Zhang, X.; Wang, S.; Lu, M.; Cui, H.; Kershaw, S. V.; Yu, W. W.; Rogach, A. L. Bright Orange Electroluminescence from Lead-Free TwoDimensional Perovskites. ACS Energy Letters 2019, 4, 242-248.

27. Hou, L.; Zhu, Y.; Zhu, J.; Li, C. Tuning Optical Properties of Lead-Free 2D Tin-Based Perovskites with Carbon Chain Spacers. Journal of Physical Chemistry C 2019, 123, $31279-31285$.

28. Lin, J. T.; Liao, C. C.; Hsu, C. S.; Chen, D. G.; Chen, H. M.; Tsai, M. K.; Chou, P. T.; Chiu, C. W. Harnessing Dielectric Confinement on Tin Perovskites to Achieve Emission Quantum Yield up to 21\%. Journal of the American Chemical Society 2019, 141, 1032410330.

29. Shi, E.; Yuan, B.; Shiring, S. B.; Gao, Y.; Guo, Y.; Su, C.; Lai, M.; Yang, P.; Kong, J.; Savoie, B. M., et al. Two-dimensional halide perovskite lateral epitaxial heterostructures. Nature 2020, 580, 614-620.

30. Wang, Z.; Wang, F.; Zhao, B.; Qu, S.; Hayat, T.; Alsaedi, A.; Sui, L.; Yuan, K.; Zhang, J.; Wei, Z.; Tan, Z. Efficient Two-Dimensional Tin Halide Perovskite Light-Emitting Diodes 
via a Spacer Cation Substitution Strategy. Journal of Physical Chemistry Letters 2020, $11,1120-1127$.

31. Nawale, V. V.; Sheikh, T.; Nag, A. Dual Excitonic Emission in Hybrid 2D Layered Tin Iodide Perovskites. Journal of Physical Chemistry C 2020, 124, 21129-21136.

32. Zhang, J.; Zhu, X.; Wang, M.; Hu, B. Establishing charge-transfer excitons in 2D perovskite heterostructures. Nature communications 2020, 11, 1-8.

33. Cheng, P.; Wu, T.; Liu, J.; Deng, W. Q.; Han, K. Lead-Free, Two-Dimensional Mixed Germanium and Tin Perovskites. Journal of Physical Chemistry Letters 2018, 9, 25182522.

34. Pecunia, V.; Occhipinti, L. G.; Chakraborty, A.; Pan, Y.; Peng, Y. Lead-free halide perovskite photovoltaics: Challenges, open questions, and opportunities. APL Materials 2020,8 .

35. Mitzi, D. B.; Feild, C.; Harrison, W.; Guloy, A. Conducting tin halides with a layered organic-based perovskite structure. Nature 1994, 369, 467-469.

36. Mitzi, D. B. Synthesis, crystal structure, and optical and thermal properties of $\left(\mathrm{C}_{4} \mathrm{H}_{9} \mathrm{NH}_{3}\right)_{2} \mathrm{MI}_{4}(\mathrm{M}=\mathrm{Ge}, \mathrm{Sn}, \mathrm{Pb})$. Chemistry of materials 1996, 8, 791-800.

37. Straus, D. B.; Kagan, C. R. Electrons, Excitons, and Phonons in Two-Dimensional Hybrid Perovskites: Connecting Structural, Optical, and Electronic Properties. Journal of Physical Chemistry Letters 2018, 9, 1434-1447.

38. Neutzner, S.; Thouin, F.; Cortecchia, D.; Petrozza, A.; Silva, C.; Srimath Kandada, A. R. Exciton-polaron spectral structures in two-dimensional hybrid lead-halide perovskites. Physical Review Materials 2018, 2, 064605.

39. Neutzner, S.; Thouin, F.; Cortecchia, D.; Petrozza, A.; Silva, C.; Srimath Kandada, A. R. 
Erratum: Exciton-polaron spectral structures in two-dimensional hybrid lead-halide perovskites [Phys. Rev. Materials 2, 064605 (2018)]. Phys. Rev. Materials 2020, 4, 059901.

40. Passarelli, J. V.; Mauck, C. M.; Winslow, S. W.; Perkinson, C. F.; Bard, J. C.; Sai, H.; Williams, K. W.; Narayanan, A.; Fairfield, D. J.; Hendricks, M. P., et al. Tunable exciton binding energy in 2D hybrid layered perovskites through donor-acceptor interactions within the organic layer. Nature chemistry 2020, 12, 672-682.

41. Calabrese, J.; Jones, N.; Harlow, R.; Herron, N.; Thorn, D.; Wang, Y. Preparation and characterization of layered lead halide compounds. Journal of the American Chemical Society 1991, 113, 2328-2330.

42. Jana, M. K.; Liu, C.; Lidin, S.; Dirkes, D. J.; You, W.; Blum, V.; Mitzi, D. B. Resolving rotational stacking disorder and electronic level alignment in a 2D oligothiophene-based lead iodide perovskite. Chemistry of Materials 2019, 31, 8523-8532.

43. Giorgi, G.; Yamashita, K.; Palummo, M. Nature of the electronic and optical excitations of ruddlesden-popper hybrid organic-inorganic perovskites: the role of the many-body interactions. The journal of physical chemistry letters 2018, 9, 5891-5896.

44. Palummo, M.; Berrios, E.; Varsano, D.; Giorgi, G. Optical properties of lead-free double perovskites by ab initio excited-state methods. ACS Energy Letters 2020, 5, 457-463.

45. Giorgi, G.; Yamashita, K.; Palummo, M. Two-dimensional optical excitations in the mixed-valence Cs 2 Au 2 I 6 fully inorganic double perovskite. Journal of Materials Chemistry C 2018, 6, 10197-10201.

46. Bernardi, M.; Ataca, C.; Palummo, M.; Grossman, J. C. Optical and Electronic Properties of Two-Dimensional Layered Materials. Nanophotonics 2017, 6, 479-493.

47. Price, M. B.; Butkus, J.; Jellicoe, T. C.; Sadhanala, A.; Briane, A.; Halpert, J. E.; Broch, K.; Hodgkiss, J. M.; Friend, R. H.; Deschler, F. Hot-carrier cooling and pho- 
toinduced refractive index changes in organic-inorganic lead halide perovskites. Nature communications 2015, 6, 1-8.

48. Thouin, F.; Neutzner, S.; Cortecchia, D.; Dragomir, V. A.; Soci, C.; Salim, T.; Lam, Y. M.; Leonelli, R.; Petrozza, A.; Srimath Kandada, A. R., et al. Stable biexcitons in two-dimensional metal-halide perovskites with strong dynamic lattice disorder. Physical Review Materials 2018, 2, 034001.

49. Thouin, F.; Srimath Kandada, A. R.; Valverde-Chávez, D. A.; Cortecchia, D.; Bargigia, I.; Petrozza, A.; Yang, X.; Bittner, E. R.; Silva, C. Electron-Phonon Couplings Inherent in Polarons Drive Exciton Dynamics in Two-Dimensional Metal-Halide Perovskites. Chemistry of Materials 2019, 31, 7085-7091.

50. Lee, J.; Koteles, E. S.; Vassell, M. Luminescence linewidths of excitons in GaAs quantum wells below 150 K. Physical Review B 1986, 33, 5512.

51. Thouin, F.; Cortecchia, D.; Petrozza, A.; Kandada, A. R. S.; Silva, C. Enhanced screening and spectral diversity in many-body elastic scattering of excitons in two-dimensional hybrid metal-halide perovskites. Physical Review Research 2019, 1, 032032.

52. Dragomir, V. A.; Neutzner, S.; Quarti, C.; Cortecchia, D.; Petrozza, A.; Roorda, S.; Beljonne, D.; Leonelli, R.; Kandada, A. R. S.; Silva, C. Lattice vibrations and dynamic disorder in two-dimensional hybrid lead-halide perovskites. arXiv preprint arXiv:1812.05255 2018,

53. Folpini, G.; Gatto, L.; Cortecchia, D.; Devetta, M.; Crippa, G.; Vozzi, C.; Stagira, S.; Petrozza, A.; Cinquanta, E. Ultrafast charge carrier dynamics in quantum confined 2D perovskite. The Journal of Chemical Physics 2020, 152, 214705.

54. Low, F. E.; Pines, D. Mobility of slow electrons in polar crystals. Physical Review 1955, 98,414 . 
55. Hendry, E.; Koeberg, M.; Bonn, M. Exciton and electron-hole plasma formation dynamics in ZnO. Physical Review B 2007, 76, 045214. 


\section{Graphical TOC Entry}

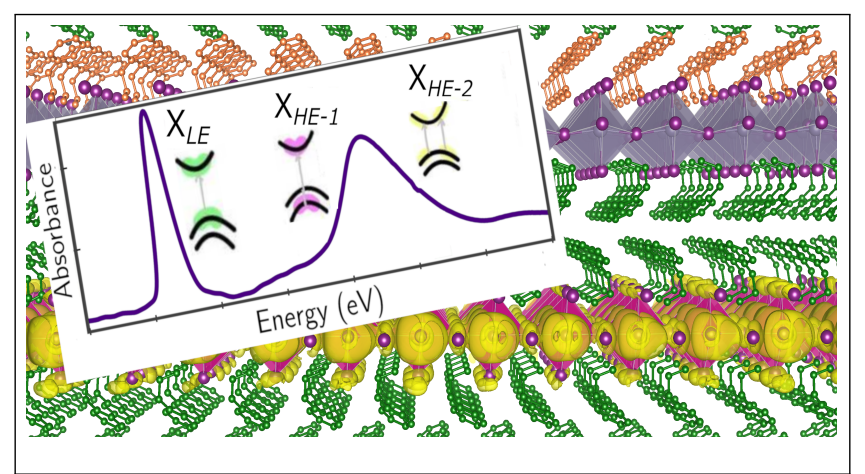

